\title{
Nuclear magnetic resonance probes for the Kondo scenario for the 0.7 feature in semiconductor quantum point contact devices
}

\author{
V Tripathi ${ }^{1}$ and N R Cooper ${ }^{2}$ \\ ${ }^{1}$ Department of Theoretical Physics, Tata Institute of Fundamental Research, \\ Homi Bhabha Road, Mumbai 400005, India \\ ${ }^{2}$ T. C. M. Group, Cavendish Laboratory, Department of Physics, University of \\ Cambridge, J. J. Thomson Avenue, Cambridge CB3 0HE, United Kingdom
}

\begin{abstract}
We discuss the expected features in nuclear relaxation and Knight shift measurements for the Kondo scenario for the " 0.7 feature" in semiconductor quantum point contact (QPC) devices defined in two-dimensional electron gases (2DEGs). As the conductance is more sensitive to the nuclear polarisation in the centre of the QPC compared to that in the 2DEG leads, our analysis is focused in the region near to the centre of the QPC. We show that the exchange coupling of a bound electron in the QPC with the nuclei would lead to, in the region near to the centre of the QPC, a much higher rate of nuclear relaxation compared to that involving exchange of nuclear spin with conduction electrons. Away from the centre of the QPC, we find that the distance beyond which the latter (conduction electron) mechanism becomes equally important is of the order of typical QPC lengths; thus, between these two electronic mechanisms, relaxation by coupling to the bound electron dominates within the QPC. Furthermore, we show that the temperature dependence of the nuclear relaxation due to coupling to the bound electron is non-monotonic as opposed to the linear- $T$ relaxation from coupling with conduction electrons. Nuclear spin diffusion processes restrict the range of validity of this analysis. We present a qualitative analysis of additional relaxation due to nuclear spin diffusion (NSD), and compare the nuclear relaxation times associated with NSD and the above electronic mechanisms. We discuss circumstances in which NSD will affect our results significantly, and suggest ways in which NSD may be suppressed in the QPC so that the Kondo physics may be unearthed. Nuclear relaxation together with Knight shift measurements, will help in verifying whether the " 0.7 " feature is indeed due to the presence of a bound electron in the QPC. While some of the results have also been discussed in the context of paramagnetic impurities in bulk conductors, our analysis is intended for application to the 0.7 effect in semiconductor systems. The qualitative and quantitative estimates we make will allow experimental tests of the Kondo scenario for the 0.7 feature in QPCs in two-dimensional electron gas heterostructures.
\end{abstract}

\section{Introduction}

\subsection{The 0.7 conductance anomaly}

The ballistic conductance $G$ of a quantum point contact (QPC) device, measured as a function of the width of the channel transverse to the current, is quantised in integer multiples of $G_{0}=2 e^{2} / h$ in the absence of a magnetic field and electron interactions. The application of a strong in-plane magnetic field lifts the electron spin degeneracy through Zeeman splitting without affecting the electron trajectories in the plane of the 
device, and the quantisation then appears in multiples of $G_{0} / 2$. These effects had been observed since 1988 [1, 2], and well-understood as arising from the quantisation of the electron momentum in the QPC in the direction transverse to the current (transverse sub-bands) [2, 3, A remarkable set of measurements [4, 5, 6, beginning in 1996, on the ubiquitous but hitherto overlooked additional " 0.7 features" between successive quantised plateaus of the ballistic conductance has, since then, lead us to critically question our understanding of electron transport in the humble QPC and directly inspired a great deal of experimental [7, 8, 9, 10, 11, 12, 13, 14, 15] and theoretical [16, 17, 18, 19, 20, 22, 21, 23, 24, 25, 26, 27, 28, 29] work.

Some of the salient features of this " 0.7 effect," as it is usually referred to are as follows. The ballistic conductance, as a function of the gate voltage (that controls the cross-sectional width of the QPC), shows shoulder-like structures at the "steps" marking the transitions between successive quantised conductance plateaus, $G_{n}=$ $n G_{0}$. The shoulders usually occur at values of around $0.7 G_{n}$ between neighbouring quantised plateaus $G_{n}$ and $G_{n+1},[4,5$ although their positions are not universal and have been known to occur as low as $0.5 G_{0}[\underline{6}, 8]$. The shoulders are not due to disorder effects nor they are transmission resonances [5]. The most prominent shoulder occurs where the QPC makes a transition from a pinch-off state $(n=0)$ to the first quantised plateau. The temperature dependence of this feature is very unusual [4, 5, 7, 8, 10]. Decreasing the temperature makes it less well-defined, and it altogether disappears at low temperatures of the order of a few tens of millikelvins. Increasing the temperature makes the feature more well-defined, until, beyond a few kelvins, the feature as well as the quantised plateaus begin to get thermally smeared out. The temperature dependence has been fitted with an Arrhenius law [7] as well as a power law [10], and the conductance change over the temperature range in which the feature exists is insufficient to resolve this ambiguity. The characteristic temperature scale associated with the feature is of the order of a kelvin. Upon the application of an in-plane magnetic field that removes electron spin degeneracy through Zeeman splitting without affecting their trajectories in the plane of the device, the 0.7 shoulder shifts lower in a smooth manner, finally moving to $0.5 G_{0}$ at fields of the order of a few tesla (corresponding to complete lifting of electron spin degeneracy). This is evidence that the feature is intimately connected with electron spin. The 0.7 feature is believed to arise due to electron interaction [4, 5, 7, 8, 10] as can be seen from the following two characteristic features. The gyromagnetic ratio $g_{e}$ of the electrons is larger in the lowest sub-bands by a factor of about two compared with the bulk GaAs value of $g_{e}=-0.44$, and decreases towards -0.44 in the higher sub-bands [4, 5]. Enhancement of the gyromagnetic ratio may be associated with electron interaction. Since in the lower sub-bands, the number of electrons in the QPC is smaller and electrostatic screening is weaker, electron interaction effects such as exchange are expected to be stronger there. In presence of a non-zero source-drain potential difference $V_{s d}, d G / d V_{s d}$ shows a zero-bias anomaly (peak) at $V_{s d}=0$, which is not generally expected for noninteracting electrons [10.

Numerous scenarios have been studied for the 0.7 feature ranging from electron spin polarisation in the QPC [4, 7, 8, 9, 16, 17, 18, 19, 20, 21], exchange splitting of few electron bound states in the QPC [22], Kondo effect arising from quasi-bound electrons in the QPC [10, 23, 24], ferromagnetic Luttinger liquids [25], charge [26] and spin density waves [27], and Wigner crystallisation effects in one dimension [20, 28]. Of these, the electron spin polarisation and Kondo scenarios have been most extensively studied, while the Wigner crystallisation scenario is a more recent proposal that also 
looks promising.

Choosing theoretically between the electron spin polarisation and Kondo pictures has proved difficult because both have been able to substantially describe the experimental observations. Recent measurements of the 0.7 feature in hole-doped GaAs in Ref. 9] used two QPCs in a hole-focusing setup that claimed to confirm the spin polarisation picture and rule out the Kondo picture as incompatible with their data. On the other hand, features such as the zero bias anomaly observed in measurements at non-zero $V_{s d}[10$, have not been explained using the spin polarisation picture, although there has been a suggestion that the zero bias anomaly can also arise from backscattering by acoustic phonons [29].

\subsection{NMR for the 0.7 feature}

In this paper we discuss the signatures in nuclear relaxation of the presence of a bound electron in a short QPC. We present a fairly detailed review on nuclear relaxation in the presence of a bound electron in the QPC. The purpose is twofold. First, these NMR methods are not yet being used in the 0.7 community and an analysis of nuclear relaxation in this context may be useful. Second, we have recently studied [30] nuclear relaxation in QPCs for the Kondo scenario as well as for the other proposed physical mechanisms for the 0.7 feature. Here we present details of the calculations for the "Kondo" part in Ref. [30, and also discuss in addition, the effects of nuclear spin diffusion processes on the relevance of the analysis.

Nuclear relaxation measurements in nanoscale systems such as QPCs have been hampered, in comparison with bulk systems, by the small number of polarised nuclei. Recently, however, it has been shown how nuclear polarisation may be created [31, 30] and detected [30, 32] in QPCs through the measurement of the two-terminal conductance. In this paper, we devote our attention to the region near the centre of the QPC as the conductance is more sensitive to nuclear polarisation in this region than it is to nuclear polarisation away from the QPC in the 2DEG leads.

We compare the nuclear relaxation rates from the coupling of the nuclei with (a) the bound electron and (b) the conduction electrons both above and below the Kondo temperature $T_{K}$. We show that near to the centre of the QPC, the relaxation through coupling with the bound electron will be in general much faster, and furthermore, follow a (very different) non-monotonous temperature dependence. In the high temperature regime $\left(T>T_{K}\right)$ the relaxation rates, respectively, due to impurity coupling, $T_{1}^{i m p}$, and conduction electrons, $T_{1}^{\text {cond-el }}$, are given by (see Eq.(26) and Eq.(29) )

$$
\begin{array}{ll}
\frac{1}{T_{1}^{i m p}}=\frac{2 A_{d}\left(\mathbf{R}_{i}\right)^{2} S(S+1)}{3 \pi \hbar\left(k_{B} T\right)\left(J \rho\left(\epsilon_{F}\right)\right)^{2}} \\
\frac{1}{T_{1}^{\text {cond-el }}}=\frac{\pi\left(k_{B} T\right)}{\hbar}\left(A_{s} \rho\left(\epsilon_{F}\right)\right)^{2} .
\end{array}
$$

Here $A_{d}\left(\mathbf{R}_{i}\right)$ is the hyperfine interaction of the nucleus at point $\mathbf{R}_{i}$ with the impurity spin $S=1 / 2$ at the origin, $A_{s}$ is the hyperfine interaction of the nucleus with the conduction electrons and $J$ is the interaction of the impurity spin and conduction electrons. $\rho\left(\epsilon_{F}\right)$ is the density of states at the Fermi energy. In the low temperature regime $\left(T<T_{K}\right)$, the relaxation rates in the two cases are (see Eq.(31) and Eq.(35))

$$
\frac{1}{T_{1}^{i m p}}=\frac{4 \pi\left(k_{B} T\right) A_{d}\left(\mathbf{R}_{i}\right)^{2}}{\hbar\left(g_{s} \mu_{B}\right)^{4}} \chi_{\mathrm{imp}}^{2},
$$




$$
\frac{1}{T_{1}^{c o n d-e l}}=\frac{\pi\left(k_{B} T\right)}{\hbar}\left(A_{s} \rho\left(\epsilon_{F}\right)\right)^{2}\left(1+\frac{2 C}{N} \frac{T_{F}}{T_{K}}\right) . \quad \text { (low temp.) }
$$

Here $\chi_{\mathrm{imp}}$ is the susceptibility of the impurity spin, $C$ is a constant of the order one, $T_{F}$ is the Fermi temperature and $N$ is the number of electrons in the QPC. The conduction electron results at low temperatures and high temperatures differ only through the enhancement of the density of states of the conduction electrons that occurs below the Kondo temperature. For details of these results we refer the reader to Sec. 3 and Sec. 4. Associating the (experimentally observed) characteristic temperature scale $\sim 1 \mathrm{~K}$ associated with the 0.7 feature with $T_{K}$, we have the following estimates. For $T=2 K$ (high temperature regime), the nuclear relaxation times associated with processes (a) and (b) near the centre of the QPC are respectively $T_{1}^{i m p} \approx 0.1 \mathrm{~s}$ and $T_{1}^{\text {cond-el }} \approx 5 \mathrm{~s}$. For $T=0.5 \mathrm{~K}$ (low temperature regime), we find $T_{1}^{i m p} \approx 3.5 \times 10^{-2} \mathrm{~S}$ and $T_{1}^{\text {cond-el }} \approx 20 \mathrm{~s}$. Away from the centre of the QPC, the nuclear relaxation rate due to impurity coupling decreases as the exchange (RKKY) interaction of the bound electron and a nuclear spin at a distance $R_{i}$ from the electron falls off as $1 /\left(k_{F} R_{i}\right)$. We show in Sec. [6 that below the Kondo temperature, relaxation by coupling to conduction electrons dominates at distances beyond $R_{i}=\left(4 \epsilon_{F} / k_{B} T_{K} k_{F}\right)$, where $\epsilon_{F}$ is the Fermi energy of the electrons in the QPC. For a 2D electron density of $10^{11} \mathrm{~cm}^{-2}$, 1D Fermi energy of $20 \mathrm{~K}$, and a Kondo temperature of $1 \mathrm{~K}$, we estimate this distance $R_{i}$ to be about $1.6 \mu \mathrm{m}$, which is of the order of the length of typical QPCs. Since nuclear relaxation in the QPC affects the conductance far more than that in the 2DEG leads, we thus conclude that between these two electronic mechanisms, the conductance is determined more by the nuclear relaxation from coupling to the impurity electron than by coupling to the conduction electrons.

The final test for a bound electron, which we propose here, comes from Knight shift measurements. The temperature dependence of the Knight shift is shown to be the same as the temperature dependence of the susceptibility of a Kondo impurity. The Knight shift may be measured by observing the conductance as a function of the frequency of an external electromagnetic wave to which the QPC is subjected. When the frequency matches the difference in energy of successive nuclear Zeeman levels, the nuclear polarisation will get destroyed resulting in a sudden change in conductance.

Internuclear dipolar interactions give rise to non-conserving spin flips and internuclear flip-flops, and limit the range of validity of our analysis. In GaAs, these interactions correspond to a field of the order of a millitesla which is equivalent to $T_{1} \sim T_{2} \sim 10^{-4} \mathrm{~s}$ in the absence of a magnetic field. However in a non-zero magnetic field of several millitesla, this intrinsic $T_{1}$ may be many orders of magnitude larger (see Sec. 7); therefore the measurements we propose should be performed in the presence of small but non-zero magnetic fields. Apart from non-conserving spin flips, internuclear spin flip-flop processes can be significant even in the presence of a magnetic field, and cause nuclear spin diffusion (NSD). Our most conservative estimate (see Sec. 7) for the nuclear spin diffusion time for the QPC is $T_{1}^{s d} \sim 0.4 \mathrm{~s}$ which is based on using the bulk value for the nuclear spin diffusion constant in GaAs. However, as we discuss later, the nuclear spin diffusion constant for a QPC with a localised electron can be much smaller than the bulk value because the resulting non-uniformity of the hyperfine interaction suppresses internuclear flip-flops. We review recent literature on NSD in quantum dots where it has been shown that NSD can be further suppressed by one to two orders of magnitude by applying fields greater than $1 \mathrm{mT}$, and also by suitable redesigning of the heterostructure as for example by growing AlGaAs layers on either 
side of the GaAs layer. We believe that the fairly long relaxation times associated with NSD in QPCs (or quantum dots) together with the possibility of further strong suppression of NSD through small magnetic fields and/or device redesigning makes it quite feasible to observe nuclear relaxation effects due to the bound electron in the QPC.

The nuclear relaxation and Knight shift measurements together enable a confirmation of the presence of a bound electron in the QPC, if any.

The rest of the paper is organised as follows. We introduce our model in Sec. 2 for a QPC with a bound electron and provide general expressions of the experimentally measured nuclear relaxation rates $T_{1}^{-1}$ and $T_{2}^{-1}$. In Sec. 3 and Sec. 4, respectively, we analyze the nuclear relaxation at temperatures above and below the Kondo temperature. The crossover between the high and low temperature regimes is discussed in Sec. 5, and Sec. 6 contains a discussion of the relative strengths of nuclear relaxation by coupling to conduction electrons and by coupling to the bound electron spin. Finally in Sec. 7 we discuss nuclear spin diffusion (NSD) effects, how it affects our earlier analysis, and ways in which NSD can be suppressed so that the Kondo scenario for the 0.7 feature may be feasibly tested with the proposed NMR method.

\section{Model}

We consider a simple model of a QPC defined in a two-dimensional electron gas (2DEG) in the $x z$ plane, taking the transport direction along the $x$ axis. Let $w_{x}, w_{z}$ be the dimensions of the QPC in the $x z$ plane, and $w_{y}$ in the direction perpendicular to the 2DEG. We assume the bound electron (impurity) of spin $\mathbf{S}$ is localised at the origin $\mathbf{r}=0$ which we take as the centre of the QPC. Let $\mathbf{I}_{i}$ be the nuclear spins of the host GaAs, and the conduction electron spin density be denoted by $\boldsymbol{\sigma}(\mathbf{r})$. The Hamiltonian is

$$
\begin{aligned}
H= & \sum_{\mathbf{k} \boldsymbol{\sigma}} \epsilon_{\mathbf{k}} c_{\mathbf{k} \boldsymbol{\sigma}}^{\dagger} c_{\mathbf{k} \boldsymbol{\sigma}}-\mathbf{H}_{0} \cdot\left(g_{s} \mu_{B} \mathbf{S}+g_{n} \mu_{n} \sum_{i} \mathbf{I}_{i}+g_{\sigma} \mu_{B} \sum_{i} \boldsymbol{\sigma}\left(\mathbf{r}_{i}\right)\right)+ \\
& +J \mathbf{S} \cdot \boldsymbol{\sigma}(0)+A_{s} \sum_{i} \mathbf{I}_{i} \cdot \boldsymbol{\sigma}\left(\mathbf{R}_{i}\right)+A_{d} \mathbf{I}_{0} \cdot \mathbf{S} .
\end{aligned}
$$

$\mathbf{H}_{0}$ is the external magnetic field. We assume that the electronic Zeeman energy $g_{\sigma} \mu_{B} \sum_{i} \mathbf{H}_{0} \cdot \boldsymbol{\sigma}\left(\mathbf{r}_{i}\right)$ is much less than the Kondo temperature associated with the (antiferromagnetic) impurity-conduction electron coupling $J,(J>0)$, such that the Kondo is not suppressed by Zeeman splitting. $A_{s}$ is the hyperfine coupling strength between the nuclei and conduction electrons. It is of the order of $100 \mu \mathrm{eV}$ per nucleus in GaAs. The hyperfine contact term $A_{d}$ coupling the impurity electron to the nuclear spins is proportional to the probability density of the localised electron wavefunction at the origin. Near to the centre of the QPC,

$$
A_{d} \approx \frac{8 A_{s}}{\left(w_{x} w_{y} w_{z}\right)} \text {. }
$$

The impurity spin is localised over a volume, typically, $w_{x} w_{y} w_{z} \sim 1 \mu \mathrm{m} \times 5 \mathrm{~nm} \times 20 \mathrm{~nm}$, that greatly exceeds the volume per nucleus $\sim 1 \mathrm{~nm}^{3}$. At temperatures much lower than the Fermi temperature, we may assume the impurity electron remains in the lowest energy state of the potential confining it. In the absence of the coupling of the impurity spin to the conduction electrons, the impurity susceptibility would have 
obeyed the Curie law. At temperatures small compared to the Fermi temperature, this susceptibility would be larger than the corresponding Pauli susceptibility of the conduction electrons.

We ignore the direct magnetic dipolar interaction of the nuclear spins. In the volume $V_{0}=w_{x} w_{y} w_{z}$ where the impurity electron is localised, we will show that the contribution to nuclear relaxation from the coupling of the nuclear spin with the conduction electrons would be small compared to the contribution from the nuclear coupling with the localised electron. The reason is that the localised electron corresponds to an enhanced spin density compared to the conduction electrons. We can also ignore the indirect exchange (RKKY) interaction of different nuclei as its strength would be small, of the order of $A_{s}^{2}$. However it is important to retain the RKKY interaction of the localised electron with distant nuclei, especially those lying outside $V_{0}$. The strength of this interaction is proportional to $J A_{s} \gg A_{s}^{2}$ (electronic energy scales such as $J$ are expected to be typically larger than corresponding nuclear energy scales such as $A_{s}$ ). The RKKY hyperfine interaction will be of the form

$$
H_{R K K Y}\left(\mathbf{R}_{i}\right)=A_{R K K Y}\left(\mathbf{R}_{i}\right) \mathbf{I}_{i} \cdot \mathbf{S},
$$

where, for $k_{F} R_{i} \gg 1$ and one spatial dimension, the RKKY interaction is [33]

$$
A_{R K K Y}\left(\mathbf{R}_{i}\right) \approx-\frac{J A_{s} \rho\left(\epsilon_{F}\right)}{V_{0}}\left[\frac{\pi}{2}-\operatorname{Si}\left(2 k_{F} R_{i}\right)\right],
$$

where $\rho\left(\epsilon_{F}\right)=4 m /\left(2 \pi \hbar^{2} k_{F} w_{y} w_{z}\right)$ is the density of electron states in the QPC and $\operatorname{Si}(x)$ is the sine integral function. At large values of its argument, $\operatorname{Si}(x) \approx$ $\pi / 2-\cos (x) / x-\sin (x) / x^{2}$, while for small values of $x, \operatorname{Si}(x) \approx x$. The hyperfine interaction $A_{d}$ for the nuclei near the centre of the QPC (given by (6) ) as well as $A_{R K K Y}\left(\mathbf{R}_{i}\right)$ for those further away can be conveniently expressed by introducing a spatially varying hyperfine coupling $A_{d}\left(\mathbf{R}_{i}\right)$ :

$$
H_{I, S}=\sum_{i} A_{d}\left(\mathbf{R}_{i}\right) \mathbf{I}_{i} \cdot \mathbf{S} .
$$

The coupling of a nuclear spin with its external environment can be written as

$$
H_{n}\left(\mathbf{R}_{i}\right)=-g_{n} \mu_{n}\left(\mathbf{H}_{0}+\mathbf{H}_{\mathrm{loc}}\left(\mathbf{R}_{i}\right)\right) \cdot \mathbf{I}_{i},
$$

where

$$
\mathbf{H}_{\mathrm{loc}}\left(\mathbf{R}_{i}\right)=-\frac{1}{g_{n} \mu_{n}}\left(A_{s} \boldsymbol{\sigma}\left(\mathbf{R}_{i}\right)+A_{d}\left(\mathbf{R}_{i}\right) \mathbf{S}\right)
$$

is the local field due to electrons at the site $\mathbf{R}_{i}$. The second contribution in (11) is more important when the impurity to host nucleus distance is not very large because the susceptibility of the localised spin, $\sim \mu_{B}^{2} / k_{B} T$ is a factor $\epsilon_{F} / k_{B} T$ larger than the Pauli susceptibility per conduction electron.

The local field is the sum of an "average" part $\left\langle\mathbf{H}_{\mathrm{loc}}\right\rangle$ and a fluctuation part $\delta \mathbf{H}_{\text {loc }}$. The nuclear resonance occurs at a frequency $\omega_{n}$ given by

$$
\hbar \omega_{n}\left(\mathbf{R}_{i}\right)=g_{n} \mu_{n} H_{0}\left(1+K\left(\mathbf{R}_{i}\right)\right),
$$

where

$$
K\left(\mathbf{R}_{i}\right)=\left\langle H_{\text {loc }}^{z}\left(\mathbf{R}_{i}\right)\right\rangle / H_{0}
$$

is the Knight shift. The Knight shift in general depends on the location $\mathbf{R}_{i}$. 
The longitudinal and transverse nuclear relaxation rates (due to local field fluctuations) $T_{\|}^{-1}$ and $T_{\perp}^{-1}$ are respectively [34]

$$
\begin{aligned}
& T_{\|}^{-1}\left(\mathbf{R}_{i}\right)=\frac{\left(g_{n} \mu_{n}\right)^{2}}{2 \hbar^{2}} \int_{-\infty}^{\infty} d t\left\langle\delta H_{\mathrm{loc}}^{z}\left(\mathbf{R}_{i}, t\right) \delta H_{\mathrm{loc}}^{z}\left(\mathbf{R}_{i}, 0\right)\right\rangle \\
& T_{\perp}^{-1}\left(\mathbf{R}_{i}\right)=\frac{\left(g_{n} \mu_{n}\right)^{2}}{4 \hbar^{2}} \int_{-\infty}^{\infty} d t e^{i \omega_{n} t}\left\langle\delta H_{\mathrm{loc}}^{+}\left(\mathbf{R}_{i}, t\right) \delta H_{\mathrm{loc}}^{-}\left(\mathbf{R}_{i}, 0\right)\right\rangle .
\end{aligned}
$$

These are related to the experimentally measured longitudinal relaxation rate $T_{1}^{-1}$ and transverse relaxation rate $T_{2}^{-1}$ through [34]

$$
\begin{aligned}
& T_{1}^{-1}=2 T_{\perp}^{-1}, \\
& T_{2}^{-1}=T_{\|}^{-1}+T_{\perp}^{-1} .
\end{aligned}
$$

Thus the Knight shifts as well as the nuclear relaxation rates depend on the locations of the nuclei.

It is possible to express the correlators of the fluctuating magnetic fields in (13) in terms of the dynamic susceptibility $\chi^{\alpha \beta}\left(\mathbf{R}_{i}, \omega\right)$ using the fluctuation-dissipation theorem. Here $\alpha$ and $\beta$ are the longitudinal $(z)$ and transverse $(+,-)$ labels. The fluctuation-dissipation theorem gives

$$
\operatorname{Im} \chi^{\alpha \beta}\left(\mathbf{R}_{i}, \omega\right)=\frac{1}{\hbar} \tanh \left(\frac{\hbar \omega}{2 k_{B} T}\right) C^{\alpha \beta}\left(\mathbf{R}_{i}, \omega\right),
$$

where

$$
C^{\alpha \beta}\left(\mathbf{R}_{i}, \omega\right)=\int_{-\infty}^{\infty} d t e^{i \omega t}\left\langle\delta M^{\alpha}\left(\mathbf{R}_{i}, t\right) \delta M^{\beta}\left(\mathbf{R}_{i}, 0\right)\right\rangle
$$

is the correlator of the fluctuations of the magnetic moment $\mathbf{M}$. At low frequencies $\omega \ll k_{B} T / \hbar$, (15) simplifies to

$$
\operatorname{Im} \frac{\chi^{\alpha \beta}\left(\mathbf{R}_{i}, \omega\right)}{\omega} \approx\left(\frac{\omega}{2 k_{B} T}\right) C^{\alpha \beta}\left(\mathbf{R}_{i}, \omega\right)
$$

We now study two extreme cases. The first concerns nuclei not very far from the impurity so that the relaxation of the nuclei is dominated by their coupling to the impurity. The second case concerns distant nuclei where the RKKY interaction is small and the nuclear relaxation is dominated by their coupling to the conduction electrons. We will study the nuclear relaxation both above and below the Kondo temperature of the impurity electron.

\section{Temperatures above $T_{K}$}

\subsection{Relaxation due to impurity coupling}

The local field at a nucleus at $\mathbf{R}_{i}$ has a simple relation with the magnetic moment $\mathbf{M}$ of the impurity electron:

$$
\mathbf{H}_{\mathrm{loc}}\left(\mathbf{R}_{i}\right) \approx-\frac{A_{d}\left(\mathbf{R}_{i}\right)}{g_{n} \mu_{n}} \mathbf{S}=-\frac{A_{d}\left(\mathbf{R}_{i}\right)}{g_{n} g_{s} \mu_{n} \mu_{s}} \mathbf{M}
$$

Using this relation between $\mathbf{M}$ and $\mathbf{H}_{\mathrm{loc}}$ together with (13) and (15), the nuclear relaxation rates at low frequencies can be shown to be

$$
T_{\|}^{-1}\left(\mathbf{R}_{i}\right)=\left.k_{B} T\left(\frac{A_{d}\left(\mathbf{R}_{i}\right)}{\hbar g_{s} \mu_{B}}\right)^{2} \operatorname{Im} \frac{\chi_{\mathrm{imp}}^{z z}(\omega)}{\omega}\right|_{\omega \rightarrow 0},
$$


and

$$
T_{\perp}^{-1}\left(\mathbf{R}_{i}\right)=\frac{1}{4 \hbar}\left(\frac{A_{d}\left(\mathbf{R}_{i}\right)}{g_{s} \mu_{B}}\right)^{2} \operatorname{coth}\left(\frac{\hbar \omega_{n}}{2 k_{B} T}\right) \operatorname{Im} \chi_{\operatorname{imp}}^{+-}\left(\omega_{n}\right)
$$

In our case, $k_{B} T$ is much larger than the nuclear Zeeman energy $\hbar \omega_{n}$, so $T_{\perp}^{-1}$ is approximately

$$
T_{\perp}^{-1}\left(\mathbf{R}_{i}\right)=\left.\frac{k_{B} T}{2}\left(\frac{A_{d}\left(\mathbf{R}_{i}\right)}{\hbar g_{s} \mu_{B}}\right)^{2} \operatorname{Im} \frac{\chi_{\mathrm{imp}}^{+-}(\omega)}{\omega}\right|_{\omega \rightarrow 0} .
$$

$\chi_{\mathrm{imp}}$ is the susceptibility of the impurity electron. We need to obtain expressions for the imaginary part of the impurity susceptibility.

Let $T_{e 1}^{-1}$ and $T_{e 2}^{-1}$ be the longitudinal and transverse relaxation times for the impurity, and let $\chi_{\mathrm{imp}}^{L}$ and $\chi_{\mathrm{imp}}^{T}$ be respectively the longitudinal and transverse static impurity susceptibilities:

$$
\begin{aligned}
\chi_{\text {imp }}^{L} & =g_{s} \mu_{B} \partial\left\langle S_{z}\right\rangle / \partial H_{0}, \\
\chi_{\text {imp }}^{T} & =g_{s} \mu_{B}\left\langle S_{z}\right\rangle / H_{0} .
\end{aligned}
$$

At small magnetic fields, there is no difference between the static longitudinal and transverse impurity susceptibility. Expressions for the imaginary part of the impurity susceptibility are available in the literature [35]:

$$
\begin{aligned}
& \operatorname{Im} \frac{\chi_{\mathrm{imp}}^{z z}(\omega)}{\omega}=\chi_{\operatorname{imp}}^{L} \frac{T_{e 1}}{1+\left(\omega T_{e 1}\right)^{2}}, \\
& \operatorname{Im} \frac{\chi_{\operatorname{imp}}^{+-}(\omega)}{2 \omega}=\chi_{\operatorname{imp}}^{T} \frac{T_{e 2}}{1+\left[\left(\omega-\omega_{e}\right) T_{e 2}\right]^{2}} .
\end{aligned}
$$

$T_{e 1}$ and $T_{e 2}$ also depend on the frequency but we are interested only in the zero frequency limits. From Ref. [34,

$$
\begin{array}{ll}
T_{e 1}^{-1}=T_{e 2}^{-1}=\frac{\pi k_{B} T}{\hbar}\left(J \rho\left(\epsilon_{F}\right)\right)^{2}, & \omega_{e} T_{e 2} \ll 1, \\
T_{e 1}^{-1}=2 T_{e 2}^{-1}=\pi S\left(J \rho\left(\epsilon_{F}\right)\right)^{2} \omega_{e}, & \omega_{e} T_{e 2} \gg 1 .
\end{array}
$$

The corresponding expressions for the imaginary part of the impurity susceptibility may obtained from (22) by substituting the values of the static transverse and longitudinal impurity susceptibility defined in (21). 34] For $\omega_{e} T_{e 2} \ll 1$ we have

$$
\begin{aligned}
& \left.\operatorname{Im} \frac{\chi_{\mathrm{imp}}^{z z}(\omega)}{\omega}\right|_{\omega \rightarrow 0}=\left.\operatorname{Im} \frac{\chi_{\mathrm{imp}}^{+-}(\omega)}{2 \omega}\right|_{\omega \rightarrow 0} \\
& =\frac{2 \hbar S(S+1)\left(g_{s} \mu_{B}\right)^{2}}{3 \pi\left(k_{B} T\right)^{2}\left(J \rho\left(\epsilon_{F}\right)\right)^{2}}, \quad \omega_{e} T_{e 2} \ll 1 ;
\end{aligned}
$$

thus the nuclear relaxation rates are

$$
T_{\|}^{-1}\left(\mathbf{R}_{i}\right)=T_{\perp}^{-1}\left(\mathbf{R}_{i}\right)=\frac{A_{d}\left(\mathbf{R}_{i}\right)^{2} S(S+1)}{3 \pi \hbar\left(k_{B} T\right)\left(J \rho\left(\epsilon_{F}\right)\right)^{2}}, \quad \omega_{e} T_{e 2} \ll 1 .
$$

For $\omega_{e} T_{e 2} \gg 1$, which is the case at low temperatures and/or high fields, the fluctuations are very anisotropic. The imaginary part of the impurity susceptibility 
and the corresponding nuclear relaxation rates are

$$
\begin{aligned}
\left.\operatorname{Im} \frac{\chi_{\mathrm{imp}}^{+-}(\omega)}{2 \omega}\right|_{\omega \rightarrow 0} & =\frac{\pi S^{2}\left(g_{s} \mu_{B}\right)^{2}\left(J \rho\left(\epsilon_{F}\right)\right)^{2}}{2 \omega_{e}^{2}}, \\
T_{\perp}^{-1}\left(\mathbf{R}_{i}\right) & =\frac{\pi\left(k_{B} T\right) A_{d}\left(\mathbf{R}_{i}\right)^{2} S^{2}\left(J \rho\left(\epsilon_{F}\right)\right)^{2}}{2 \hbar^{3} \omega_{e}^{2}},
\end{aligned}
$$

and

$$
\begin{aligned}
\left.\operatorname{Im} \frac{\chi_{\mathrm{imp}}^{z z}(\omega)}{\omega}\right|_{\omega \rightarrow 0} & =\frac{\left(g_{s} \mu_{B}\right)^{2} e^{-\hbar \omega_{e} / k_{B} T}}{\pi \omega_{e} S\left(k_{B} T\right)\left(J \rho\left(\epsilon_{F}\right)\right)^{2}}, \\
T_{\|}^{-1}\left(\mathbf{R}_{i}\right) & =\frac{A_{d}\left(\mathbf{R}_{i}\right)^{2} e^{-\hbar \omega_{e} / k_{B} T}}{\pi \hbar^{2} \omega_{e}\left(J \rho\left(\epsilon_{F}\right)\right)^{2}} \approx 0, \quad \omega_{e} T_{e 2} \gg 1 .
\end{aligned}
$$

The experimentally observed relaxation rates $T_{1}^{-1}$ and $T_{2}^{-1}$ are obtained by using the relations in (14).

\subsection{Relaxation due to conduction electron coupling}

Expressions for nuclear relaxation due to coupling to conduction electrons can be obtained by substituting $J, \omega_{e}$ and $T_{e}$ in (23) by $A_{s}, \omega_{n}$ and $T_{n}$. Since the nuclear Zeeman energy is so small, we will always be interested in the high temperature case. The result is [36]

$$
T_{1}^{-1}=T_{2}^{-1}=\frac{\pi\left(k_{B} T\right)}{\hbar}\left(A_{s} \rho\left(\epsilon_{F}\right)\right)^{2} .
$$

Note that the nuclear relaxation due to coupling to the impurity spin does not have a Korringa-like temperature dependence. This may be regarded as a signature of the presence of a localised electron.

\section{Temperatures below $T_{K}$}

Let us now consider nuclear relaxation below the Kondo temperature $T_{K}$. The more interesting case, again, is that of relaxation by coupling to the impurity spin.

\subsection{Relaxation due to impurity spin}

The following analysis presumes that $g_{s} \mu_{B} H_{0} / k_{B} T_{K} \ll 1$. For higher fields, the analysis of Sec. 3 should be used. At small fields, we have mentioned earlier that there is no difference between the static longitudinal and transverse susceptibilities. When $T \ll T_{K}$, the imaginary part of the susceptibility satisfies an elegant relation, [37]

$$
\left.\operatorname{Im} \frac{\chi_{\mathrm{imp}}^{z z}(\omega)}{\omega}\right|_{\omega \rightarrow 0}=\left.\operatorname{Im} \frac{\chi_{\mathrm{imp}}^{+-}(\omega)}{2 \omega}\right|_{\omega \rightarrow 0}=\frac{2 \pi \hbar \chi_{\mathrm{imp}}^{2}}{\left(g_{s} \mu_{B}\right)^{2}} .
$$

As a result, the nuclear relaxation rates take the simple form

$$
T_{\|}^{-1}=T_{\perp}^{-1}=\frac{2 \pi\left(k_{B} T\right) A_{d}\left(\mathbf{R}_{i}\right)^{2}}{\hbar\left(g_{s} \mu_{B}\right)^{4}} \chi_{\mathrm{imp}}^{2} .
$$


Using (17) in the definition of the Knight shift, (12), it is easy to see that

$$
K\left(\mathbf{R}_{i}\right)=\frac{A_{d}\left(\mathbf{R}_{i}\right) \operatorname{Re} \chi_{\mathrm{imp}}^{z z}(0)}{\left(g_{n} \mu_{n}\right)\left(g_{s} \mu_{B}\right)} .
$$

$\operatorname{Re} \chi^{z z}(0)$ is just the static impurity susceptibility $\chi_{\mathrm{imp}}$. Eq.(32) is also valid above the Kondo temperature. Different nuclei will couple with the impurity with different strengths $A_{d}\left(\mathbf{R}_{i}\right)$; however, the temperature dependence of the Knight shift will be the same. Since $A_{d}\left(\mathbf{R}_{i}\right)$ falls off with distance, one would observe a spread of Knight shifts and the spread would continuously increase in the same sense as the impurity susceptibility as the temperature is lowered. Ultimately, the susceptibility will saturate at the lowest temperatures which would correspond to a maximum spread of the Knight shifts. The same can be said for the relaxation rates (see (31)). Such behaviour of the Knight shift has been reported in $\mathrm{Cu}$ :Fe alloys [38].

Combining (31) and (32) we get [34, 37]

$$
K\left(\mathbf{R}_{i}\right)^{2} T_{1}\left(\mathbf{R}_{i}\right) T=\frac{\left(g_{s} \mu_{B}\right)^{2}}{\left(g_{n} \mu_{n}\right)^{2}} \frac{\hbar}{4 \pi k_{B}} .
$$

Eq. (33) has the form of Korringa relaxation.36

\subsection{Relaxation due to conduction electron coupling}

Relaxation due to coupling to conduction electrons matters only for those nuclei that are so far from the impurity that their RKKY coupling to the impurity is weaker than their hyperfine coupling with the conduction electrons. That happens when $k_{F} R_{i} \gg 1$. As the temperature falls below the impurity Kondo temperature, there is an enhancement in the density of states at the Fermi energy: [39]

$$
\tilde{\rho}\left(\epsilon_{F}\right)=\rho\left(\epsilon_{F}\right)\left[1+C\left(T_{F} / T_{K}\right) 1 / N\right], \quad T \ll T_{K}
$$

where the tilde denotes the Kondo-enhanced density of states at the Fermi energy, $C$ is a constant of order one, and $N$ is the number of electrons in the QPC. This leads to an enhancement of the relaxation rate [40] given in (29):

$$
T_{1}^{-1}=\frac{\pi\left(k_{B} T\right)}{\hbar}\left(A_{s} \tilde{\rho}\left(\epsilon_{F}\right)\right)^{2}, \quad T \ll T_{K} .
$$

Thus we can summarise,

$$
\frac{\left.T_{1}^{-1}\right|_{T \ll T_{K}}}{\left.T_{1}^{-1}\right|_{T \gg T_{K}}}=\frac{\tilde{\rho}\left(\epsilon_{F}\right)^{2}}{\rho\left(\epsilon_{F}\right)^{2}} \approx 1+2 C\left(T_{F} / T_{K}\right) 1 / N .
$$

We should perhaps use this enhanced density of states even for the case of relaxation through coupling to the impurity spin below the Kondo temperature.

Application of a magnetic field will tend to decrease the density of states towards the high temperature value. In the Kondo regime, the impurity susceptibility is proportional to the density of states of the conduction electrons. From the known Bethe ansatz solution for the impurity magnetisation, we can extract the magnetic field dependence of the density of states: [41]

$$
\tilde{\rho}\left(\epsilon_{F}, H_{0}\right) \approx \rho\left(\epsilon_{F}\right)\left[1+\frac{C T_{F}}{N T_{K}}\left(1-C^{\prime}\left(\frac{g_{s} \mu_{B} H_{0}}{k_{B} T_{K}}\right)^{2}\right)\right],
$$

where $C^{\prime}$ is a constant of order one. 


\section{Crossover between high and low temperature regimes}

We have two independent parameters demarcating low and high temperature behaviour: $\omega_{e} T_{e 2}$ and $T / T_{K}$. So we need to discuss further the meaning of low and high temperature regimes.

The Kondo temperature is approximately $T_{K} \approx \epsilon_{F} e^{-1 / J \rho\left(\epsilon_{F}\right)}$, where $J \rho\left(\epsilon_{F}\right)$ is the unrenormalised, i.e., bare, Kondo coupling. Given that $\epsilon_{F} \approx 20 \mathrm{~K}$, we cannot have too small a value for $J \rho\left(\epsilon_{F}\right)$ if we are to have any hope of probing the behaviour on either side of the Kondo temperature. Even for $J \rho\left(\epsilon_{F}\right)=0.1$, we would get a very small $T_{K} \approx 10^{-3} \mathrm{~K}$. Let us therefore assume that the bare $J \rho\left(\epsilon_{F}\right) \lesssim 1$.

In our discussion of the behaviour above $T_{K}$, we had obtained two regimes depending on the magnitude of $\omega_{e} T_{e 2}$. A small value of $\omega_{e} T_{e 2}$ corresponded to a high temperature. From (23), we can see that the criterion for high temperature behaviour (26) is

$$
T \gg T_{\text {high }}=\frac{\hbar \omega_{e}}{\pi k_{B}\left(J \rho\left(\epsilon_{F}\right)\right)^{2}} .
$$

This is not too different from the temperature corresponding to the Zeeman splitting of the localised electron given our expectations regarding the value of $J \rho\left(\epsilon_{F}\right)$. Now the Kondo temperature can either be larger or smaller than $T_{\text {high }}$.

Suppose $T_{K} \ll T_{\text {high }}$. Then in principle we have three regimes: $T \gg T_{\text {high }}$, $T_{K} \ll T \ll T_{\text {high }}$, and $T \ll T_{K}$. In the high temperature regime, $T \gg T_{\text {high }}$, we will observe a non-Korringa relaxation, (26), due to coupling with the impurity spin.

Note that the condition $T_{K} \ll T_{\text {high }}$ corresponds to $T_{K} \ll g_{s} \mu_{B} H_{0} / k_{B}$. However all our discussion of $T \ll T_{K}$ assumed that the Zeeman splitting of the impurity was less than the Kondo temperature. We should not use those results for $T \ll T_{K}$. In fact, the large Zeeman field suppresses the "Fermi liquid" regime of the Kondo model. Thus there is no Kondo regime for $T_{K} \ll T_{\text {high }}$. There are just two regimes separated by $T_{\text {high }}$, and the relaxation rates in these two regimes are given by (26), (27) and (28). The maximum relaxation rate occurs around $T_{\text {high }}$ where $\omega_{e} T_{e 2} \approx 1$.

Suppose $T_{K} \gg T_{\mathrm{high}}$. If the impurity Zeeman splitting is small, then this is the likely scenario. In that case we should redefine our high temperature regime to mean $T \gg T_{K}$. Owing to the qualitative change in the susceptibility and other properties at $T<T_{K}$, we must not use (when $T<T_{K}$ ) (26), (27) and (28) which were derived assuming a Curie susceptibility for the impurity spin and the bare value of the dimensionless Kondo coupling. Such assumptions are correct only when $T \gg T_{K}$. In the low temperature regime, $T \ll T_{K}$, the relaxation will be given by (31). In the region of $T=T_{K}$, the ratio of the relaxation rate on the high temperature side to the Kondo side is of the order of $1 /\left(J \rho\left(\epsilon_{F}\right)\right)^{2}$. Since the coupling constant $J \rho\left(\epsilon_{F}\right)$ diverges below $T=T_{K}$, the Kondo relaxation rate will dominate near $T=T_{K}$ and below. As the temperature is decreased starting from the high temperature side, one would observe a steady enhancement of the relaxation rate (obeying the $1 / T$ law) up to $T \sim T_{K}$, followed by a linear- $T$ decrease according to (31). (Maximum relaxation rate at $T \approx T_{K}$.)

Further confirmation of the Kondo effect can be made by measuring the temperature dependence of the Knight shift as shown in (32). If the temperature dependence of the Knight shift is the same as that of the Kondo impurity susceptibility both above and below the Kondo temperature, then the Kondo effect will be confirmed. 


\section{Relaxation by impurity coupling and conduction electron coupling}

Let us compare the relative magnitudes of relaxation by coupling to the impurity spin and to conduction electrons. Consider the low temperature regime, $T \ll T_{K}$, and a small magnetic field such that $T_{K} \gg g_{s} \mu_{B} H_{0} / k_{B}$. Thus we need to compare the relaxation rates in (31) and (35). First consider nuclei inside the region $V_{0}$ about the impurity. In this region, we have mentioned earlier that $A_{d}\left(\mathbf{R}_{i}\right) \approx$ $8 A_{s} /\left(w_{x} w_{y} w_{z}\right)$. It is easy to see that the ratio of the relaxation rates through coupling with the impurity and with the conduction electrons is of the order of $\left(A_{d}\left(\mathbf{R}_{i}\right) \chi_{\mathrm{imp}} /\left(g_{s} \mu_{B}\right)^{2}\right)^{2} /\left(A_{s} \rho\left(\epsilon_{F}\right)\right)^{2} \sim\left(4 \pi \hbar^{2} k_{F} / m w_{x} k_{B} T_{K}\right)^{2}$, where we used $\rho\left(\epsilon_{F}\right)=$ $4 m /\left(2 \pi \hbar^{2} k_{F} w_{y} w_{z}\right)$. Estimating $2 \pi / w_{x} \sim k_{F}$, the ratio works out to $\sim\left(4 \epsilon_{F} / T_{K}\right)^{2} \gg 1$. Therefore in the region $V_{0}$ around the impurity electron, nuclear relaxation is primarily through coupling with this electron. Outside $V_{0}$, the impurity RKKY coupling decreases as $1 /\left(k_{F} R_{i}\right)$. The distance at which relaxation by conduction electrons becomes comparable depends on the strength of $J \rho\left(\epsilon_{F}\right)$. We have argued before that we need $J \rho\left(\epsilon_{F}\right) \lesssim 1$ in order to have any chance of measuring on both sides of the Kondo temperature with the usual apparatus. Thus the RKKY interaction is smaller than $A_{s}$ by a factor of $1 /\left(k_{F} R_{i}\right)$. Therefore the distance beyond which relaxation is mostly by conduction electron coupling corresponds to $\left(4 \epsilon_{F} / k_{B} T_{K}\right)^{2} /\left(k_{F} R_{i}\right)^{2}<1$, or $R_{i}>4 \epsilon_{F} /\left(k_{B} T_{K} k_{F}\right)$.

The Kondo impurity, if present, will be easier to detect through its direct or RKKY exchange coupling with the nuclear spins for three reasons. First, we have already seen above that the higher susceptibility of the impurity compared to the conduction electron susceptibility for $T \ll T_{F}$ leads to a stronger nuclear relaxation rate. Second, the temperature dependence of the nuclear relaxation in the former case does not follow the Korringa law at high temperatures. Third, the Knight shift will broaden as the temperature is lowered, and the temperature dependence of the broadening will be directly proportional to the Kondo impurity susceptibility (which is well-known). All cases we discussed obey the Korringa law at temperatures below the Kondo temperature.

We have not discussed the role of possible electron-electron interaction. Electron interaction will affect both the density of states as well as the impurity susceptibility. Proximity to a ferromagnetic instability of the conduction electrons will enhance the impurity susceptibility (through enhancement of the electron gyromagnetic ratio) which will tend to increase the relaxation rate. However one needs to keep in mind any interaction effects on the density of states. In the absence of the Kondo impurity, Moriya has shown that the nuclear relaxation rate is enhanced by electron-electron repulsion [42].

\section{Relaxation by nuclear spin diffusion}

In our treatment we have so far ignored internuclear dipolar interactions that will cause internuclear flip-flops and nonconserving nuclear spin flips. In GaAs, the intrinsic nuclear relaxation times $T_{1}$ and $T_{2}$ can roughly estimated to be of the order of

$\hbar / \epsilon_{d d} \sim 10^{-4} \mathrm{~s}$, where $\epsilon_{d d}$ is the magnetic dipolar interaction of neighbouring nuclei corresponding to a field of about $1 \mathrm{mT}$ acting on the nuclei. In non-zero fields, however, $T_{1}$ can be larger by several orders of magnitude as for example has been observed [43] in GaAs where $T_{1} \sim 10^{3} \mathrm{~s}$ at fields of about $140 \mathrm{mT}$. In the following discussion we assume that a field of several millitesla is present so that nonconserving spin flips due 
to internuclear interaction may be ignored.

In addition to nonconserving spin flips, one also has internuclear spin flip-flop processes. The latter give rise to nuclear spin diffusion (NSD) and occur even in the presence of an external magnetic field. NSD effects in quantum dots are a topic of much recent study owing to their importance for nuclear spin polarisation based qubits. A thorough analysis of NSD is not attempted here given the incomplete understanding in the literature of the same on quantum dots. Instead we discuss qualitatively the conditions under which NSD effects can be important in our case, and how this may be suppressed to allow the electronic relaxation mechanisms to have a greater effect on the QPC conductance. A simple model for studying the spatial dependence and temporal decay of the nuclear polarisation is

$$
\frac{\partial M}{\partial t}=D \nabla^{2} M-\frac{M-M_{0}}{T_{1}(\mathbf{r})}
$$

where $D$ is the nuclear spin diffusion constant and $M_{0}$ is the steady state nuclear polarisation in the given external magnetic field. The nuclear spin diffusion constant is related to the decoherence time $T_{2}$ for the nuclear polarisation; for a cubic lattice such as GaAs [44, 45],

$$
D \approx \frac{a^{2}}{30 T_{2}}
$$

where $a$ is the nearest distance between nuclei of the same species. In pure, bulk GaAs, the internuclear flip-flop processes set an upper limit to $T_{2} \sim \hbar / \epsilon_{d d} \sim 10^{-4} \mathrm{~s}$, which gives us $D_{\text {bulk }} \sim 10^{-13} \mathrm{~cm}^{2} / \mathrm{s}$. Experimentally observed values of the nuclear spin diffusion constant in bulk GaAs due to internuclear dipolar interactions are in agreement with this rough estimate [46].

In a quantum dot with a localised impurity electron, the spatial variation of the localised electron wavefunction leads to a spatially varying hyperfine contact interaction. This affects both the relaxation and spatial distribution of the nuclear polarisation. First, the spatial variation of the hyperfine interaction in the quantum dot has been shown [47 to cause a suppression of the diffusion constant $D_{d o t}$ in the dot by a factor of the order of 10 compared to $D_{b u l k}$ because nuclear flip-flop transitions in this case do not conserve energy. Experimentally, the NSD constant in quantum dots has also been reported to be small compared to the bulk value [48, 49]. Second, during the build-up of the nuclear polarisation, the inhomogeneity of the hyperfine interaction translates into an inhomogeneous nuclear polarisation, with a maximum near the centre of the dot, and rapid decay outside the dot. Due to the presence of the diffusion term, the solution of Eq.(39) with a nonuniform initial distribution of nuclear polarisation does not in general decay exponentially with time [50]. Exponential decay can however take place if the diffusion energy in Eq.(39) is smaller than $\hbar / T_{1}$. We estimate the nuclear diffusion rate $1 / T_{1}^{s d}$ to be the order of $D_{d o t} / l_{\min }^{2}$, where $l_{\min }$ is the smallest dimension of the QPC along which nuclear spins may diffuse. In our case, $l_{\min }=w_{y}=5 \mathrm{~nm}$, and conservatively using for $D_{d o t}$ the bulk diffusion value $D_{b u l k}$ for GaAs, we find $T_{1}^{s d} \approx 0.4 \mathrm{~s}$. If we take into account the suppression of the diffusion constant in the quantum dot because of an inhomogeneous hyperfine interaction [47, we will have $T_{1}^{s d} \sim 4 \mathrm{~s}$ for $D_{d o t} \sim 0.1 D_{b u l k}$. In recent measurements on quantum dots [51, enhancement of the nuclear relaxation time by a factor of nearly two orders of magnitude (to nearly 100s) has been reported at fields more than $1 \mathrm{mT}$. Another way to increase the NSD time is by designing the 2DEG such that we have AlGaAs layers on either side of the 2DEG, instead of on one side as we have considered here. 
NSD is suppressed in a direction perpendicular to the 2DEG because of the change of material from GaAs to AlGaAs as well as disorder in AlGaAs 52. In such a redesigned QPC, we should regard the transverse width $w_{z}=20 \mathrm{~nm}$ as $l_{\min }$, and that will give $T_{1}^{s d} \sim 6$.4s even if inhomogeneous hyperfine interaction effects are not taken into account, and $T_{1}^{s d} \sim 65 \mathrm{~s}$ if this is taken into account. We note that in experiments on quantum dots in Ref. [53, $T_{1}^{\text {sd }}$ has been estimated to be as long as 200s.

To compare with the nuclear relaxation rates in the Kondo scenario which is the subject of this paper, we have for the QPC $A_{d} \approx 5.8 \times 10^{-29} \mathrm{~J}$ per nucleus and we associate the experimental energy scale determining the conductance with the Kondo temperature: $T_{K} \approx 1 \mathrm{~K}$. For a QPC defined in a GaAs 2DEG with conduction electron density $10^{11} \mathrm{~cm}^{-2}$, the $1 \mathrm{D}$ Fermi energy $\epsilon_{F}\left(m=0.067 m_{e}\right)$ in the lowest sub-band is about $20 \mathrm{~K}$; and using $T_{K} \approx \epsilon_{F} e^{-1 / J \rho\left(\epsilon_{F}\right)}$, we estimate the bare (high temperature) value of $J \rho\left(\epsilon_{F}\right) \approx 0.35$. In the "high" temperature region $\left(T>T_{K}\right)$, say $T=2 \mathrm{~K}$, Eq.(26) then gives the relaxation time due to coupling to the impurity electron as $T_{1}^{i m p} \approx 0.1 \mathrm{~s}$. This is comparable with our most conservative estimate above for the relaxation time due to nuclear spin diffusion, while if we take into account the suppression of NSD due to inhomogeneous hyperfine interaction, and/or design the 2DEG to suppress diffusion perpendicular to the 2DEG, NSD effects are much smaller and may be ignored in a first treatment. The relaxation time using the above parameters due to coupling to conduction electrons as estimated from Eq.(29) is $T_{1}^{\text {cond-el }} \approx 5 \mathrm{~s}$, which is also long compared to relaxation by coupling to the paramagnetic impurity. In the "low" temperature region $\left(T<T_{K}\right)$, the relaxation time associated with coupling to the paramagnetic impurity as given by Eq.(31) (using $\left.\chi_{\mathrm{imp}} \approx\left(g_{s} \mu_{B}\right)^{2} / k_{B} T_{K}\right)$ is $T_{1}^{i m p} \approx 3.5 \times 10^{-2} \mathrm{~s}$ at $T=0.5 \mathrm{~K}$, which is much shorter than the relaxation times $T_{1}^{s d} \sim 10 \mathrm{~s}$ and $T_{1}^{\text {cond-el }}$ (at this temperature $T_{1}^{\text {cond-el }} \approx 20 \mathrm{~s}$ ) respectively due to NSD and coupling to conduction electrons. The latter two effects are therefore safely ignored in the QPC, except at very low temperatures when NSD may dominate because it does not vanish at $T=0$. Away from the centre of the QPC, relaxation by coupling to the paramagnetic impurities and coupling to conduction electrons become comparable. We estimate this distance from the discussion in Sec. 6 to be $R_{i}=\left(4 \epsilon_{F} / k_{B} T_{K} k_{F}\right) \approx 1.6 \mu \mathrm{m}$, which is of the order of the length of the QPC. Thus outside the QPC, relaxation by coupling to conduction electrons is also important. It is easily seen that the same is also true for NSD. Nevertheless, since the conductance is very sensitive to the Overhauser field in the QPC and not to the Overhauser field in the 2DEG, we conclude that to a first approximation, $T_{1}$ obtained from the conductance of the QPC is dominated by the coupling to the paramagnetic impurity compared to nuclear spin diffusion and coupling to conduction electrons.

To summarise, nuclear spin diffusion effects may be ignored in our analysis if the experiments are performed in fields of several millitesla, and the temperature is high enough such that the nuclear diffusion time $l_{\min }^{2} / D$ is much longer than the relaxation time $T_{1}$ from electronic processes. A more accurate treatment of NSD effects is needed at very low temperatures and for long QPCs.

V.T. thanks the support of TIFR and a DST Ramanujan Fellowship [sanction no. $100 /$ IFD $/ 154 / 2007-08]$. N.R.C. acknowledges support by EPSRC grant $\mathrm{GR} / \mathrm{S} 61263 / 01$.

[1] D. A. Wharam, T. J. Thornton, R. Newbury, M. Pepper, H. Ahmed, J. E. F. Frost, D. G. Hasko, D. C. Peacock, D. A. Ritchie, and G. A. C. Jones, J. Phys. C: Sol. St. Phys. 21, L209 (1988).

[2] B. J. van Wees, H. van Houten, C. W. J. Beenakker, J. G. Williamson, L. P. Kouwenhoven, D. van der Marel, and C. T. Foxon, Phys. Rev. Lett. 60, 848 (1988). 
[3] L. I. Glazman, G. B. Lesovik, D. E. Khmelnitskii, and R. I. Shekhter, Pis'ma Zh. Éksp. Teor. Fiz. 48, 218 (1988); [JETP Lett. 48, 238 (1988)].

[4] K. J. Thomas, J. T. Nicholls, M. Y. Simmons, M. Pepper, D. R. Mace, and D. A. Ritchie, Phys. Rev. Lett. 77, 135 (1996).

[5] K. J. Thomas, J. T. Nicholls, N. J. Appleyard, M. Y. Simmons, M. Pepper, D. R. Mace, W. R. Tribe, and D. A. Ritchie, Phys. Rev. B 58, 4846 (1998).

[6] K. J. Thomas, J. T. Nicholls, M. Pepper, W. R. Tribe, M. Y. Simmons, and D. A. Ritchie, Phys. Rev. B 61, R13365 - R13368 (2000).

[7] A. Kristensen, H. Bruus, A. E. Hansen, J. B. Jensen, P. E. Lindelof, C. J. Marckmann, J. Nygård, C. B. Sørensen, F. Beuscher, A. Forchel, and M. Michel, Phys. Rev. B 62, 10950 (2000).

[8] D. J. Reilly, G. R. Facer, A. S. Dzurak, B. E. Kane, R. G. Clark, P. J. Stiles, R. G. Clark, A. R. Hamilton, J. L. O'Brien, N. E. Lumpkin, L. N. Pfeiffer, and K. W. West, Phys. Rev. B 63, 121311 (2001).

[9] L. P. Rokhinson, L. N. Pfeiffer, and K. W. West, Phys. Rev. Lett. 96, 156602 (2006).

[10] S. M. Cronenwett, H. J. Lynch, D. Goldhaber-Gordon, L. P. Kouwenhoven, C. M. Marcus, K. Hirose, N. S. Wingreen, and V. Umansky, Phys. Rev. Lett. 88, 226805 (2002).

[11] A. C. Graham, K. J. Thomas, M. Pepper, N. R. Cooper, M. Y. Simmons, and D. A. Ritchie, Phys. Rev. Lett. 91, 136404 (2003).

[12] A. C. Graham, M. Pepper, M. Y. Simmons, and D. A. Ritchie, Phys. Rev. B 72, 193305 (2005); A. C. Graham, D. L. Sawkey, M. Pepper, M. Y. Simmons, and D. A. Ritchie, Phys. Rev. B 75, 035331 (2007).

[13] R. de Picciotto, L. N. Pfeiffer, K. W. Baldwin, and K. W. West, Phys. Rev. Lett. 92, 036805 (2004).

[14] N. J. Appleyard, J. T. Nicholls, M. Pepper, W. R. Tribe, M. Y. Simmons, and D. A. Ritchie, Phys. Rev. B 62, R16275 (2000).

[15] P. Roche, J. Ségala, D. C. Glattli, J. T. Nicholls, M. Pepper, A. C. Graham, K. J. Thomas, M. Y. Simmons, and D. A. Ritchie, Phys. Rev. Lett. 93, 116602 (2004); L. DiCarlo, Y. Zhang, D. T. McClure, D. J. Reilly, C. M. Marcus, L. N. Pfeiffer, and K. W. West, Phys. Rev. Lett. 97, $036810(2006)$.

[16] C.-K. Wang and K.-F. Berggren, Phys. Rev. B 54, R14257 (1996); Phys. Rev. B 57, 4552 (1998); K.-F. Berggren and I. I. Yakimenko, Phys. Rev. B 66, 085323 (2002); Anton A. Starikov, I. I. Yakimenko, and K.-F. Berggren, Phys. Rev. B 67, 235319 (2003).

[17] I. A. Shelykh, N. T. Bagraev, V. K. Ivanov, and L. E. Klyachkin, Semiconductors 36, 65 (2002); N. T. Bagraev, I. A. Shelykh, V. K. Ivanov, and L. E. Klyachkin, Phys. Rev. B 70, 155315 (2004).

[18] P. Havu, M. J. Puska, R. M. Nieminen, and V. Havu, Phys. Rev. B 70, 233308 (2004).

[19] B. Spivak and F. Zhou, Phys. Rev. B 61, 16730 (2000).

[20] A. D. Klironomos, J. S. Meyer, and K. A. Matveev, Europhys. Lett. 74, 679 (2006).

[21] D. J. Reilly, Phys. Rev. B 72, 033309 (2005).

[22] V. V. Flambaum and M. Yu. Kuchiev, Phys. Rev. B 61, R7869 (2000); T. Rejec, A. Ramsak, and J. H. Jefferson, J. Phys.: Condens. Matt. 12, L233 (2000); I. A. Shelykh, N. G. Galkin, and N. T. Bagraev, Phys. Rev. B 74, 085322 (2006).

[23] Y. Meir, K. Hirose, and N. S. Wingreen, Phys. Rev. Lett. 89, 196802 (2002); K. Hirose, Y. Meir, and N. S. Wingreen, Phys. Rev. Lett. 90, 026804 (2003); A. Golub, T. Aono, and Y. Meir, Phys. Rev. Lett. 97, 186801 (2006); T. Rejec and Y. Meir, Nature 442, 900 (2006).

[24] P. S Cornaglia, C. A Balseiro, Europhys. Lett. 67634 (2004).

[25] L. Bartosch, M. Kollar, and P. Kopietz, Phys. Rev. B 67, 092403 (2003); K. Yang, Phys. Rev. Lett. 93, 066401 (2004). B. Spivak and F. Zhou, Phys. Rev. B 61, 16730 (2000).

[26] O. P. Sushkov, Phys. Rev. B 64, 155319 (2001).

[27] S. M. Reimann, M. Koskinen, and M. Manninen, Phys. Rev. B 59, 1613 (1999).

[28] K. A. Matveev, Phys. Rev. Lett. 92, 106801 (2004); Phys. Rev. B 70, 245319 (2004); A. D. Klironomos, R. R. Ramazashvili, and K. A. Matveev, Phys. Rev. B 72, 195343 (2005).

[29] G. Seelig and K. A. Matveev, Phys. Rev. Lett. 90, 176804 (2003).

[30] N. R. Cooper and V. Tripathi, arXiv:0710.4302 [accepted to Phys. Rev. B]

[31] V. Tripathi, A. C. H. Cheung, and N. R. Cooper, Europhys. Lett. 81, 68001 (2008).

[32] J. A. Nesteroff et al., Phys. Rev. Lett. 93, 126601 (2004).

[33] Y. Yafet, Phys. Rev. B 36, 3948 (1987).

[34] A. Narath, Physica Scripta 11, 237 (1975).

[35] W. Götze and P. Wölfle, J. Low Temp. Phys. 5, 575 (1971).

[36] J. Korringa, Physica 16, 601 (1950). 
[37] H. Shiba, Prog. Theor. Phys. 54, 967 (1975).

[38] H. Alloul, Physica 86-88B, 449 (1977).

[39] P. Nozières, J. Low Temp. Phys. 17, 31 (1974).

[40] W. A. Roshen and W. F. Saam, Phys. Rev. B 22, 5495 (1980).

[41] W. A. Roshen and W. F. Saam, Phys. Rev. B 26, 2644 (1982).

[42] T. Moriya, J. Phys. Soc. Jap. 18, 516 (1963).

[43] J. A. McNeil and W. G. Clark, Phys. Rev. B 13, 4705 (1976).

[44] G. R. Khutsishvili, Sov. Phys. Uspekhi 8, 743 (1966).

[45] N. Bloembergen, Physica 15, 386 (1949).

[46] D. Paget, Phys. Rev. B 25, 4444 (1982).

[47] C. Deng and X. Hu, Phys. Rev. B 72, 165333 (2005).

[48] V. Bayot, E. Grivei, J.-M. Beuken, S. Melinte and M. Shayegan, Phys. Rev. Lett. 79, 1718 (1997).

[49] J. Stephens, J. Berezowsky, R. K. Kawakami, A. C. Gossard and D. D. Awschalom, Appl. Phys. Lett. 92, 037205 (2004).

[50] I. Tifrea and M. E. Flatte, Phys. Rev. Lett. 90, 237601 (2003).

[51] P. Maletinsky, A. Badolato and A. Imamoglu, Phys. Rev. Lett. 99, 056804 (2007).

[52] A. Malinowski and R. T. Harley, Sol. St. Comm. 114, 419 (2000).

[53] K. Ono and S. Tarucha, Phys. Rev. Lett. 92, 256803 (2004). 